\title{
Algebraic Properties of Toeplitz Operators on the Pluriharmonic Bergman Space
}

\author{
Jingyu Yang, ${ }^{1,2}$ Liu Liu, ${ }^{1}$ and Yufeng Lu ${ }^{1}$ \\ ${ }^{1}$ School of Mathematical Sciences, Dalian University of Technology, Dalian 116024, China \\ ${ }^{2}$ School of Mathematics and Statistics, Chifeng University, Chifeng 024000, China \\ Correspondence should be addressed to Jingyu Yang; yjy923@163.com
}

Received 20 June 2012; Revised 7 September 2012; Accepted 6 October 2012

Academic Editor: Nikolai M. Vasilevski

Copyright (c) 2013 Jingyu Yang et al. This is an open access article distributed under the Creative Commons Attribution License, which permits unrestricted use, distribution, and reproduction in any medium, provided the original work is properly cited.

\begin{abstract}
We study some algebraic properties of Toeplitz operators with radial or quasihomogeneous symbols on the pluriharmonic Bergman space. We first give the necessary and sufficient conditions for the product of two Toeplitz operators with radial symbols to be a Toeplitz operator and discuss the zero-product problem for several Toeplitz operators with radial symbols. Next, we study the finite-rank product problem of several Toeplitz operators with quasihomogeneous symbols. Finally, we also investigate finite rank commutators and semicommutators of two Toeplitz operators with quasihomogeneous symbols.
\end{abstract}

\section{Introduction}

Let $d v$ denote the normalized Lebesgue volume measure on the unit ball $B_{n}$ of $C^{n} . L^{2}\left(B_{n}, d v\right)$ is the Hilbert space of Lebesgue square integrable functions on $B_{n}$ with the inner product:

$$
\langle f, g\rangle=\int_{B_{n}} f(z) \overline{g(z)} d v(z) .
$$

The Bergman space $L_{a}^{2}\left(B_{n}\right)$ is the Hilbert space consisting of all holomorphic functions on $B_{n}$ which are also in $L^{2}\left(B_{n}, d v\right)$. It is well known that $L_{a}^{2}\left(B_{n}\right)$ is a reproducing function space with reproducing kernel:

$$
K_{z}(w)=\frac{1}{(1-\langle w, z\rangle)^{n+1}}, \quad z, w \in B_{n} .
$$

The pluriharmonic Bergman space $L_{h}^{2}\left(B_{n}\right)$ is the closed subspace of $L^{2}\left(B_{n}, d v\right)$ consisting of the pluriharmonic functions on $B_{n}$. It is easy to verify that

$$
L_{h}^{2}\left(B_{n}\right)=L_{a}^{2}\left(B_{n}\right)+\overline{L_{a}^{2}\left(B_{n}\right)},
$$

where $\overline{L_{a}^{2}\left(B_{n}\right)}=\left\{\bar{f} \mid f \in L_{a}^{2}\left(B_{n}\right), f(0)=0\right\}$. Then $L_{h}^{2}\left(B_{n}\right)$ is also a reproducing function space with reproducing kernel:

$$
R_{z}(w)=K_{z}(w)+\overline{K_{z}(w)}-1, \quad z, w \in B_{n} .
$$

Let $Q$ denote the orthogonal projection from $L^{2}\left(B_{n}, d v\right)$ onto $L_{h}^{2}\left(B_{n}\right)$, then

$$
Q \varphi(z)=\left\langle\varphi, R_{z}\right\rangle, \quad \varphi \in L^{2}\left(B_{n}, d v\right) .
$$

Let $P$ be the orthogonal projection from $L^{2}\left(B_{n}, d v\right)$ onto $L_{a}^{2}\left(B_{n}\right)$, then

$$
P \varphi(z)=\left\langle\varphi, K_{z}\right\rangle, \quad \varphi \in L^{2}\left(B_{n}, d v\right) .
$$

By (4), we have

$$
\mathrm{Q} \varphi(z)=P \varphi(z)+\overline{P(\bar{\varphi})(z)}-P \varphi(0) .
$$

For a function $\varphi \in L^{\infty}\left(B_{n}, d v\right)$, the Toeplitz operator $T_{\varphi}$ : $L_{h}^{2}\left(B_{n}\right) \rightarrow L_{h}^{2}\left(B_{n}\right)$ with symbol $\varphi$ is defined by

$$
T_{\varphi}(f)=Q(\varphi f)(z)=\int_{B_{n}} f(w) \varphi(w) \overline{R_{z}(w)} d v(w) .
$$

In the setting of the classical Hardy space, for $f$ and $g$ in $L^{\infty}(T)$, Brown and Halmos [1] proved that $T_{f} T_{g}=T_{h}$ if and only if one of the following conditions holds: (a) $g$ is analytic, (b) $\bar{f}$ is analytic. They also showed that, in both cases $h=f g$. On the Bergman space of the unit disk, conditions (a) and (b) 
are only sufficient but not necessary. Ahern and Čučković [2] showed that a Brown-Halmos-type result holds for Toeplitz operators with harmonic symbols under certain conditions. Later, Ahern gave the necessary and sufficient conditions for the product of two Toeplitz operators with harmonic symbols to be a Toeplitz operator in [3]. For general symbols, the product problem for Toeplitz operators remains open. Louhichi et al. [4] gave the necessary and sufficient conditions for the product of two quasihomogeneous Toeplitz operators to be a Toeplitz operator.

On the Hardy or Bergman space of several complex variables, the situation is much more complicated. Ding [5] characterized when the product of two Toeplitz operators with bounded symbols on the Hardy space $H^{2}\left(D^{n}\right)$ is still a Toeplitz operator. Motivated by the results of Ahern and Čučković [2], Choe et al. solved the product problem for Toeplitz operators with pluriharmonic symbols on the Bergman space of the polydisk [6]. On the Bergman space of the unit ball, Zhou and Dong [7] gave the necessary and sufficient conditions for the product of two Toeplitz operators with radial symbols to be equal to a Toeplitz operator. In [8], they only gave the necessary condition for the product problem of two separately quasihomogeneous Toeplitz operators. Recently, Lu and Zhang characterized when the product of two quasihomogeneous Toeplitz operators is equal to another quasihomogeneous Toeplitz operator in [9].

For the so-called "zero-product" problem, Brown and Halmos [1] easily deduced that if $T_{f} T_{g}=0$ on the Hardy space $H^{2}(T)$ then either $f$ or $g$ must be identically zero. A natural and interesting problem on the classical Hardy space of one complex variable is the following zero-product problem: If $T_{\varphi_{1}} T_{\varphi_{2}} \cdots T_{\varphi_{n}}=0$, whether one of these symbols is identically zero. Guo [10] proved that if $T_{\varphi_{1}} T_{\varphi_{2}} \cdots T_{\varphi_{5}}=0$, then there exists some $i$ such that $\varphi_{i}=0$. In [11], Gu showed that $T_{\varphi_{1}} T_{\varphi_{2}} \cdots T_{\varphi_{6}}=0$, then there exists some $i$ such that $\varphi_{i}=0$. Aleman and Vukotić [12] completely solved the zero-product problem for several Toeplitz operators on the Hardy space. In [2], Ahern and Čučković proved that the result is analogous to that in [1] for two Toeplitz operators with harmonic symbols on the Bergman space of unit disk. Moreover in [13] they proved that if $T_{f} T_{g}=0$, where $f$ is arbitrary bounded and $g$ is radial, then either $f \equiv 0$ or $g \equiv 0$. Čučković and Louhichi [14] studied finite rank product of several quasihomogeneous Toeplitz operators on the Bergman space of the unit disk. On the Bergman space of the unit ball, Choe and Koo [15] gave a result which is analogous to that in [2] with an assumption about the continuity of the symbols on an open subset of the boundary and solved the zero-product problem for several Toeplitz operators with harmonic symbols that have Lipschitz continuous extensions to the whole boundary. Also, analogous results were proved on polydisk in [16]. Recently, those zero product results have been generalized to finite rank product results in [17]. Le [18] discussed finite rank products of several Toeplitz operators on the weighted Bergman space of the unit ball. Dong and Zhou [8] investigated the zeroproduct problem of two Toeplitz operators, one of whose symbols is separately quasihomogeneous and the other is arbitrary bounded. In [7] they studied the zero-product problem for several Toeplitz operators with radial symbols. Ding [5] considered the problem of two Toeplitz operators with symbols in $L^{2}\left(T^{n}\right)$ on the Hardy space of the polydisk.

For two Toeplitz operators $T_{\varphi}$ and $T_{\psi}$ we define the commutator and semicommutator, respectively, by

$$
\begin{gathered}
{\left[T_{\varphi}, T_{\psi}\right]=T_{\varphi} T_{\psi}-T_{\psi} T_{\varphi},} \\
\left(T_{\varphi}, T_{\psi}\right]=T_{\varphi \psi}-T_{\varphi} T_{\psi} .
\end{gathered}
$$

On the Hardy space the problem of determining when the commutator or semicommutator has finite rank has been completely solved (see $[19,20]$ ). For the Bergman space the problem seems to be far from solution. Guo et al. [21] completely characterized the finite rank commutator and semicommutator of two Toeplitz operators with bounded harmonic symbols on the Bergman space of the unit disk. Luecking [22] showed that finite rank Toeplitz operators on the Bergman space of the unit disk must be zero. Čučković and Louhichi [14] studied the finite rank semi-commutators and commutators of Toeplitz operators with quasihomogeneous symbols on the Bergman space of the unit disk and obtained different results from the case of harmonic Toeplitz operators. Lu and Zhang $[9,23]$ characterized finite rank commutators and semicommutators of two quasihomogeneous Toeplitz operators on the Bergman space of the unit ball and the polydisk, respectively.

The fact that the product of two harmonic functions is no longer harmonic adds some mystery in the study of operators on the harmonic Bergman space. The theory of Toeplitz operators on the harmonic Bergman space is quite different from that on $L_{a}^{2}$. For example, Choe and Lee [24] showed that two analytic Toeplitz operators on $L_{h}^{2}$ commute only when their symbols and the constant function 1 are linearly dependent, but analytic Toeplitz operators always commute on $L_{a}^{2}$. The following question was raised by Choe and Lee in [24]: If an analytic Toeplitz operator and a coanalytic Toeplitz operator on the harmonic Bergman space commute, then is one of their symbols a constant? To solve this problem, they proved in [25] that if $f, g \in H^{\infty}$ and suppose one of them is noncyclic, then $T_{f} T_{\bar{g}}=T_{\bar{g}} T_{f}$ if and only if either $f$ or $g$ is constant. On the pluriharmonic Bergman space of the unit ball, Lee and Zhu [26] characterized commuting Toeplitz operators with holomorphic symbols and obtained the necessary and sufficient condition for the product of two Toeplitz operators with pluriharmonic symbols to be equal to a Teoplitz operator. Furthermore, they gave a complete description of holomorphic symbols for which the associated Toeplitz operators have zero semicommutator. On the pluriharmonic Bergman space of the polydisk, Choe and Nam [27] obtained results which are parallel to those of [26]. Dong and Zhou [28] studied the problem of when the product of two Toeplitz operators with quasihomogeneous symbols is a Toeplitz operator on the harmonic Bergman space of the unit disk.

Motivated by the recent work of Čučković, Louhichi [14], Zhou and Dong [7, 28], and Lee and Zhu [26] we study Toeplitz operators with radial or quasihomogeneous symbols on the pluriharmonic Bergman space of the unit ball. 
The present paper is assembled as follows: In Section 2, we introduce some basic properties of the Mellin transform and Mellin convolution. In Section 3, we first give the necessary and sufficient condition for the product of two Toeplitz operators with radial symbols to be a Toeplitz operator. Then we investigate the zero-product problem for several Toeplitz operators with radial symbols. In Section 4, we study the finite rank product problem for several Toeplitz operator with quasihomogeneous symbols. In Sections 5 and 6, we discuss the finite rank commutators and semicommutators of two Toeplitz operators with quasihomogeneous symbols on the pluriharmonic Bergman space.

\section{The Mellin Transform and Mellin Convolution}

One of the most useful tools in the following calculations is the Mellin transform. The Mellin transform $\widehat{\varphi}$ of a function $\varphi \in L^{1}([0,1], r d r)$ is defined by

$$
\widehat{\varphi}(z)=\int_{0}^{1} \varphi(s) s^{z-1} d s .
$$

It is clear that $\widehat{\varphi}$ is well defined on the right half-plane $\{z$ : $\operatorname{Re} z \geq 2\}$ and analytic on $\{z: \operatorname{Re} z>2\}$. It is important and helpful to know that the Mellin transform $\widehat{\varphi}$ is uniquely determined by its value on an arithmetic sequence of integers. In fact, we have the following classical theorem [29, page 102].

Theorem 1. Suppose that $f$ is a bounded analytic function on $\{z: \operatorname{Re} z>0\}$ which vanishes at the pairwise distinct points $z_{1}, z_{2}, \ldots$, where

(1) $\inf \left\{\left|z_{n}\right|\right\}>0$ and

(2) $\sum_{n \geq 1} \operatorname{Re}\left(1 / z_{n}\right)=\infty$.

Then $f$ vanishes identically on $\{z: \operatorname{Re} z>0\}$.

Remark 2. We will often use this theorem to show that if $\varphi \in$ $L^{1}([0,1], r d r)$ and if there exists a sequence $\left(n_{k}\right)_{k \geq 0} \subset \mathbb{N}$ such that

$$
\widehat{\varphi}\left(n_{k}\right)=0, \quad \sum_{k \geq 0} \frac{1}{n_{k}}=\infty,
$$

then $\widehat{\varphi}(z)=0$ for all $z \in\{z: \operatorname{Re}(z)>2\}$ and $\operatorname{so} \varphi=0$.

In the following discussion we need a known fact about the Mellin convolution of two functions. If $f$ and $g$ are defined on $[0,1)$, then their Mellin convolution is defined by

$$
\left(f *_{M} g\right)(r)=\int_{r}^{1} f\left(\frac{r}{t}\right) g(t) \frac{d t}{t}, \quad 0 \leq r<1 .
$$

The Mellin convolution theorem states that

$$
\widehat{f *_{M} g}(s)=\widehat{f}(s) \widehat{g}(s),
$$

and that, if $f$ and $g$ are in $L^{1}([0,1], r d r)$ then so is $f *_{M} g$.

\section{Products of Toeplitz Operators with Radial Symbols}

For any multi-index $\alpha=\left(\alpha_{1}, \ldots, \alpha_{n}\right)$, where each $\alpha_{i}$ is a nonnegative integer, we write $|\alpha|=\alpha_{1}+\cdots+\alpha_{n}, \alpha$ ! $=$ $\alpha_{1} ! \cdots \alpha_{n}$ ! and $z^{\alpha}=z_{1}^{\alpha_{1}} \cdots z_{n}^{\alpha_{n}}, z=\left(z_{1}, \ldots, z_{n}\right) \in B_{n}$.

For two multi-indexes $\alpha=\left(\alpha_{1}, \ldots, \alpha_{n}\right)$ and $\beta=$ $\left(\beta_{1}, \ldots, \beta_{n}\right)$, the notation $\alpha \geq \beta$ means that $\alpha_{i} \geq \beta_{i}, i=$ $1,2, \ldots n$, and $\alpha \perp \beta$ means that $\alpha_{1} \beta_{1}+\cdots+\alpha_{n} \beta_{n}=0$. We also define $\alpha-\beta=\left(\alpha_{1}-\beta_{1}, \ldots, \alpha_{n}-\beta_{n}\right)$. Moreover, if $|\alpha-\beta|=|\alpha|-|\beta|$ if $\alpha \geq \beta$.

It is known that $\varphi$ is radial if and only if $\varphi(U z)=\varphi(z)$ for any unitary transformation $U$ of $C^{n}$. That is, $\varphi(z)$ only depends on $|z|$. Then for each radial function $\varphi$, we define $\widetilde{\varphi}$ on $[0,1)$ by $\widetilde{\varphi}(r)=\varphi(r \xi)$ where $\xi$ is a unit vector in $C^{n}$. It is obvious that $\widetilde{\varphi}$ is welldefined. In the following, we will identify an integrable radial function $\varphi$ on the unit ball with the corresponding function $\widetilde{\varphi}$ defined on the interval $[0,1)$.

Lemma 3. Let $\varphi \in L^{2}\left(B_{n}, d v\right), T_{\varphi}$ is a Toeplitz operator densely defined on $L_{h}^{2}\left(B_{n}\right)$, then the symbol map $\varphi \rightarrow T_{\varphi}$ is one to one.

Proof. Assume $T_{\varphi}=0$, we have $Q[\varphi f]=0$ for all $f \in L_{h}^{2}\left(B_{n}\right)$.

Since $z^{\beta} \in L_{h}^{2}\left(B_{n}\right)$, then we can imply that $\left\langle\varphi z^{\alpha}, z^{\beta}\right\rangle=$ 0 , which is equivalent to $\int_{B_{n}} \varphi z^{\alpha} \bar{z}^{\beta} d v(z)=0$. Since $\left\{z^{\alpha} \bar{z}^{\beta}: \alpha, \beta\right.$ are any multi-indexes $\}$ is dense in $L_{2}\left(B_{n}, d v\right)$, so we get $\varphi=0$.

A direct calculation gives the following lemma, which will be used constantly in this paper.

Lemma 4. Let $\psi$ be an integrable radial function on $B_{n}$, such that $T_{\psi}$ is a bounded operator, then for any multi-index $\alpha$,

$$
\begin{aligned}
& T_{\psi}\left(z^{\alpha}\right)=2(n+|\alpha|) \widehat{\psi}(2 n+2|\alpha|) z^{\alpha}, \\
& T_{\psi}\left(\bar{z}^{\alpha}\right)=2(n+|\alpha|) \widehat{\psi}(2 n+2|\alpha|) \bar{z}^{\alpha} .
\end{aligned}
$$

Proof. For any multi-index $\beta$, we have

$$
\begin{aligned}
\left\langle T_{\psi} z^{\alpha}, z^{\beta}\right\rangle & =\left\langle\psi z^{\alpha}, z^{\beta}\right\rangle \\
& = \begin{cases}0 & \alpha \neq \beta ; \\
\frac{2 n ! \alpha !}{(n-1+|\alpha|) !} \widehat{\psi}(2 n+2|\alpha|) & \alpha=\beta,\end{cases} \\
\left\langle z^{\alpha}, z^{\beta}\right\rangle & = \begin{cases}0 & \alpha \neq \beta ; \\
\frac{n ! \alpha !}{(n+|\alpha|) !} & \alpha=\beta,\end{cases}
\end{aligned}
$$

which implies

$$
\left\langle T_{\psi} z^{\alpha}, z^{\beta}\right\rangle=2(n+|\alpha|) \widehat{\psi}(2 n+2|\alpha|)\left\langle z^{\alpha}, z^{\beta}\right\rangle \text {. }
$$
that

If $\beta>0$, we have $\left\langle T_{\psi} z^{\alpha}, \bar{z}^{\beta}\right\rangle=0=\left\langle z^{\alpha}, \bar{z}^{\beta}\right\rangle$. This shows

$$
\left\langle T_{\psi} z^{\alpha}, \bar{z}^{\beta}\right\rangle=\left\langle z^{\alpha}, \bar{z}^{\beta}\right\rangle \quad \forall \beta>0 .
$$


Since $\left\{z^{\alpha}\right\}_{\alpha \succeq 0} \bigcup\left\{\bar{z}^{\alpha}\right\}_{\alpha \succ 0}$ is a basis for the pluriharmonic Bergman space, we have

$$
T_{\psi}\left(z^{\alpha}\right)=2(n+|\alpha|) \widehat{\psi}(2 n+2|\alpha|) z^{\alpha}
$$

By a similar argument, we have $T_{\psi}\left(\bar{z}^{\alpha}\right)=2(n+|\alpha|) \widehat{\psi}(2 n+$ $2|\alpha|) \bar{z}^{\alpha}$.

The following theorem is very simple but essential.

Theorem 5. Let $\varphi \in L^{2}\left(B_{n}, d v\right)$. Then the following assertions are equivalent.

(1) For each multi-index $\alpha$, there exists $\lambda_{|\alpha|} \in C$ which depends only on $|\alpha|$ such that $T_{\varphi}\left(z^{\alpha}\right)=\lambda_{|\alpha|} z^{\alpha}$, $T_{\varphi}\left(\bar{z}^{\alpha}\right)=\lambda_{|\alpha|} \bar{z}^{\alpha}$

(2) $\varphi$ is a radial function.

Proof. The implication $(2) \Rightarrow(1)$ is obvious fir Lemma 4 .

Now, consider the converse implication (1) $\Rightarrow(2)$. By Lemma 3, we just need to prove $T_{\varphi \circ U}=T_{\varphi}$ for any unitary transformation $U$ of $C^{n}$

First assume $T_{\varphi}\left(z^{\alpha}\right)=\lambda_{|\alpha|} z^{\alpha}$. For any unitary transformation $U$ of $C^{n}$ and $z \in B_{n}$, we have $U^{-1} z \in B_{n}$. Thus,

$$
T_{\varphi}\left(U^{-1} z\right)^{\alpha}=\lambda_{|\alpha|}\left(U^{-1} z\right)^{\alpha}
$$

By (7), we have

$$
T_{\varphi \circ U}\left(z^{\alpha}\right)=P\left[\varphi(U z) z^{\alpha}\right]+\overline{P\left[\overline{\varphi(U z)} \bar{z}^{\alpha}\right]}-P\left[\varphi(U z) z^{\alpha}\right](0) .
$$

A direct calculation shows that

$$
\begin{aligned}
P\left[\varphi(U z) z^{\alpha}\right](w) & =\int_{B^{n}} \frac{\varphi(U z) z^{\alpha}}{(1-\langle w, z\rangle)^{n+1}} d v(z) \\
& =\int_{B^{n}} \frac{\varphi(z)\left(U^{-1} z\right)^{\alpha}}{\left(1-\left\langle w, U^{-1} z\right\rangle\right)^{n+1}} d v(z) \\
& =\int_{B^{n}} \frac{\varphi(z)\left(U^{-1} z\right)^{\alpha}}{(1-\langle U w, z\rangle)^{n+1}} d v(z) \\
& =P\left[\varphi(z)\left(U^{-1} z\right)^{\alpha}\right](U w) .
\end{aligned}
$$

Similarly, we have

$$
\begin{gathered}
P\left[\overline{\varphi(U z)} \overline{z^{\alpha}}\right](w)=P\left[\overline{\varphi(z)} \overline{\left(U^{-1} z\right)^{\alpha}}\right](U w), \\
P\left[\varphi(U z) z^{\alpha}\right](0)=P\left[\varphi(z)\left(U^{-1} z\right)^{\alpha}\right](0) .
\end{gathered}
$$

Then we can imply

$$
\begin{aligned}
T_{\varphi \circ U}\left(z^{\alpha}\right)(w)= & P\left[\varphi(z)\left(U^{-1} z\right)^{\alpha}\right](U w) \\
& +P\left[\overline{\varphi(z)\left(U^{-1} z\right)^{\alpha}}\right](U w) \\
& -P\left[\varphi(z)\left(U^{-1} z\right)^{\alpha}\right](0) \\
= & T_{\varphi}\left(U^{-1} z\right)^{\alpha}(U w) \\
= & {\left[\lambda_{|\alpha|}\left(U^{-1} z\right)^{\alpha}\right](U w) } \\
= & \lambda_{|\alpha|} w^{\alpha} \\
= & T_{\varphi}\left(z^{\alpha}\right)(w) .
\end{aligned}
$$

So $T_{\varphi \circ U}\left(z^{\alpha}\right)=T_{\varphi}\left(z^{\alpha}\right)$. With the same method, we get $T_{\varphi \circ U}\left(\bar{z}^{\alpha}\right)=T_{\varphi}\left(\bar{z}^{\alpha}\right)$. Therefore, we can imply $T_{\varphi \circ U}=T_{\varphi}$ on $L_{h}^{2}\left(B_{n}\right)$. By Lemma 3 we obtain that $\varphi \circ U=\varphi$ and $\varphi$ is a radial function.

Corollary 6. Let $\varphi_{1}$ and $\varphi_{2}$ be two square integrable radial functions on $B_{n}$ such that $T_{\varphi_{1}}$ and $T_{\varphi_{2}}$ are bounded operators. If $T_{\varphi_{1}} T_{\varphi_{2}}=T_{\psi}$, then $\psi$ is a radial function.

Proof. According to (14) we have

$$
\begin{aligned}
& T_{\varphi_{1}} T_{\varphi_{2}}\left(z^{\alpha}\right)=(2 n+2|\alpha|)^{2} \widehat{\varphi}_{1}(2 n+2|\alpha|) \widehat{\varphi}_{2}(2 n+2|\alpha|) z^{\alpha}, \\
& T_{\varphi_{1}} T_{\varphi_{2}}\left(\bar{z}^{\alpha}\right)=(2 n+2|\alpha|)^{2} \widehat{\varphi}_{1}(2 n+2|\alpha|) \widehat{\varphi}_{2}(2 n+2|\alpha|) \bar{z}^{\alpha} .
\end{aligned}
$$

It follows from Theorem 5 that $\psi$ is a radial function.

Theorem 7. Let $\varphi_{1}$ and $\varphi_{2}$ be two square integrable radial functions on $B_{n}$, such that $T_{\varphi_{1}}$ and $T_{\varphi_{2}}$ are bounded operators. Then $T_{\varphi_{1}} T_{\varphi_{2}}$ is equal to the Toeplitz operator $T_{\psi}$ if and only if $\psi$ is a solution of the equation

$$
1 *_{M} \psi=\varphi_{1} *_{M} \varphi_{2}
$$

where 1 denotes the constant function with value one.

Proof. For each multi-index $\alpha$, it follows from (14) that

$$
T_{\varphi_{1}} T_{\varphi_{2}}\left(z^{\alpha}\right)=T_{\psi}\left(z^{\alpha}\right), \quad T_{\varphi_{1}} T_{\varphi_{2}}\left(\bar{z}^{\alpha}\right)=T_{\psi}\left(\bar{z}^{\alpha}\right)
$$

are equivalent to

$$
\frac{1}{2 n+2|\alpha|} \widehat{\psi}(2 n+2|\alpha|)=\widehat{\varphi}_{1}(2 n+2|\alpha|) \widehat{\varphi}_{2}(2 n+2|\alpha|) \text {. }
$$

A direct calculation shows $\widehat{1}(2 n+2|\alpha|)=1 /(2 n+2|\alpha|)$, so (27) is equivalent to

$$
\widehat{1 *_{M} \psi}(2 n+2|\alpha|)=\widehat{\varphi_{1} *_{M} \varphi_{2}}(2 n+2|\alpha|) .
$$

By Remark 2, we obtain that (28) is equivalent to (25). 
Example 8. Let $l$ and $m$ greater than or equal to -1 . Then

$$
T_{|z|^{\mid}} T_{|z|^{m}}= \begin{cases}\frac{l}{l-m} T_{|z|^{l}}-\frac{m}{l-m} T_{|z|^{m}} & l \neq m \\ T_{|z|^{l}(1+l \ln |z|)} & l=m .\end{cases}
$$

In [18] Le discussed finite rank product of Toeplitz operators with general symbols on the weighted Bergman space of the unit ball. Similarly, we will use Mellin transform and Remark 2 to study zero-product problem of Toeplitz operators with radial symbols on pluriharmonic Bergman space.

Theorem 9. Let $\varphi_{i}$ be integrable radial functions on $B_{n}$, such that $T_{\varphi_{i}}$ be bounded operators, $i=1, \ldots, N$. If $T_{\varphi_{1}} \cdots T_{\varphi_{N}}=0$, then $\varphi_{i}=0$ for some $i$.

Proof. Suppose $T_{\varphi_{1}} \cdots T_{\varphi_{N}}=0$, then for any multi-index $\alpha$, we have

$$
T_{\varphi_{1}} \cdots T_{\varphi_{N}}\left(z^{\alpha}\right)=0 \quad T_{\varphi_{1}} \cdots T_{\varphi_{N}}\left(\bar{z}^{\alpha}\right)=0 .
$$

Using (14), we have

$$
\widehat{\varphi}_{1}(2 n+2|\alpha|) \cdots \widehat{\varphi}_{N}(2 n+2|\alpha|)=0
$$

Let $E_{i}=\left\{|\alpha| \in \mathbb{N}: \widehat{\varphi}_{i}(2 n+2|\alpha|)=0\right\}$. Notice that $E_{1} \cup \cdots \cup$ $E_{N}=\mathbb{N}$, hence there exists some $i$ such that $\sum_{|\alpha| \in E_{i}}(1 /|\alpha|)=$ $\infty$, then by Remark $2 \varphi_{i}=0$.

Corollary 10. Let $\varphi$ be an integrable radial function on $B_{n}$, such that $T_{\varphi}$ is a bounded operator. Then $T_{\varphi}^{2}=T_{\varphi}$ if and only if either $\varphi=0$ or $\varphi=1$.

Proof. If $T_{\varphi}^{2}=T_{\varphi}$, then $T_{\varphi} T_{\varphi-1}=0$. By Theorem 9 we have $\varphi=0$ or $\varphi=1$. The converse is clear.

\section{Product of $n$ Toeplitz Operator}

In this section, we will show that if the product of several quasihomogeneous Toeplitz operators has finite rank, then at least one of the symbols is equal to zero. This result is analogous to Theorem 3.2 in [18], but we get it in a different way.

Definition 11. Let $p, s \geq 0$. A function $f \in L^{1}\left(B_{n}, d v\right)$ is called a quasihomogeneous function of degree $(p, s)$ if $f$ is of the form $\xi^{p} \bar{\xi}^{s} \varphi$, where $\varphi$ is a radial function, that is,

$$
f(r \xi)=\xi^{p} \bar{\xi}^{s} \varphi(r),
$$

for any $\xi$ in the unit sphere $S_{n}$ and $r \in[0,1)$.
Lemma 12. Let $p, s$ be two multi-indexes and let $\varphi$ be an integrable radial function on $B_{n}$, such that $T_{\xi^{p} \varphi}, T_{\bar{\xi}^{p} \varphi}$, and $T_{\xi^{p} \bar{\xi}^{s} \varphi}$ are bounded operators. Then for any multi-index $\alpha$,

$$
\begin{aligned}
& T_{\xi^{p} \bar{\xi}^{s} \varphi}\left(z^{\alpha}\right) \\
& = \begin{cases}\frac{2(p+\alpha) !(n+|p|+|\alpha|-|s|) !}{(p+\alpha-s) !(n-1+|\alpha|+|p|) !} & \\
\quad \times \widehat{\varphi}(2 n+2|\alpha|+|p|-|s|) z^{p+\alpha-s} & p+\alpha \succeq s, \\
\frac{2 s !(n+|s|-|\alpha|-|p|) !}{(s-\alpha-p) !(n-1+|s|) !} & \\
\times \widehat{\varphi}(2 n+|s|-|p|) \bar{z}^{s-\alpha-p} & p+\alpha \preceq s, \\
0 & p+\alpha \geq s, \\
& p+\alpha \Varangle s,\end{cases}
\end{aligned}
$$

$$
T_{\xi^{p} \bar{\xi}_{\varphi}^{s}}\left(\bar{z}^{\alpha}\right)
$$

$$
\begin{cases}\frac{2(s+\alpha) !(n+|s|+|\alpha|-|p|) !}{(s+\alpha-p) !(n-1+|\alpha|+|s|) !} & \\ \quad \times \widehat{\varphi}(2 n+2|\alpha|+|s|-|p|) \bar{z}^{s+\alpha-p} & s+\alpha \geq p, \\ \frac{2 p !(n+|p|-|\alpha|-|s|) !}{(p-\alpha-s) !(n-1+|p|) !} & \\ \quad \times \widehat{\varphi}(2 n+|p|-|s|) z^{p-\alpha-s} & s+\alpha \preceq p, \\ 0 & s+\alpha \geq p, \\ & s+\alpha \npreceq p .\end{cases}
$$

Especially, if $p, s$ are two nonzero multi-indexes with $p \perp s$, one has

$$
\begin{aligned}
& T_{\xi^{p} \bar{\xi}_{\varphi}^{s}}\left(z^{\alpha}\right) \\
& =\left\{\begin{array}{cc}
\frac{2(p+\alpha) !(n+|p|+|\alpha|-|s|) !}{(p+\alpha-s) !(n-1+|\alpha|+|p|) !} & \alpha \geq s, \\
\quad \times \widehat{\varphi}(2 n+2|\alpha|+|p|-|s|) z^{p+\alpha-s} & \alpha \geq s . \\
0 & \alpha
\end{array}\right.
\end{aligned}
$$

$$
\begin{aligned}
& T_{\xi^{p} \bar{\xi}^{s} \varphi}\left(\bar{z}^{\alpha}\right) \\
& =\left\{\begin{array}{cc}
\frac{2(s+\alpha) !(n+|s|+|\alpha|-|p|) !}{(s+\alpha-p) !(n-1+|\alpha|+|s|) !} & \\
\quad \times \widehat{\varphi}(2 n+2|\alpha|+|s|-|p|) \bar{z}^{s+\alpha-p} & \alpha \geq p, \\
0 & \alpha \geq p .
\end{array}\right.
\end{aligned}
$$

Proof. Here we only give the proof of the first equation. For multi-indexes $\alpha$ and $\beta$, 


$$
\begin{aligned}
& \left\langle P\left[\xi^{p} \bar{\xi}^{s} \varphi z^{\alpha}\right], z^{\beta}\right\rangle \\
& \quad=\int_{B_{n}} \xi^{p} \bar{\xi}^{s} \varphi(z) z^{\alpha} \bar{z}^{\beta} d v(z) \\
& \quad=\int_{[0,1)}^{2 n r^{2 n+|\alpha|+|\beta|-1} \varphi(r) d r \int_{S_{n}} \xi^{p} \bar{\xi}^{s} \xi^{\alpha} \bar{\xi}^{\beta} d \sigma(\xi)} \\
& \quad=\left\{\begin{array}{lc}
\frac{2 n !(p+\alpha) !}{(n-1+|\alpha|+|p|) !} & p+\alpha \geq s, \\
\times \widehat{\varphi}(2 n+2|\alpha|+|p|-|s|) & \beta=p+\alpha-s, \\
0 & p+\alpha \geq s, \\
0 & p+\alpha \geq s . \alpha-s,
\end{array}\right.
\end{aligned}
$$

If $p+\alpha \succeq s$, we have

$$
\begin{aligned}
\left\langle z^{p+\alpha-s}, z^{\beta}\right\rangle= & \int_{B_{n}} z^{p+\alpha-s} \bar{z}^{\beta} d v(z) \\
= & \int_{[0,1)} 2 n r^{2 n+|p|+|\alpha|-|s|+|\beta|-1} d r \\
& \times \int_{S_{n}} \xi^{p+\alpha-s} \bar{\xi}^{\beta} d \sigma(\xi) \\
= & \begin{cases}\frac{n !(p+\alpha-s) !}{(n+|\alpha|+|p|-|s|) !} & \beta=p+\alpha-s, \\
0 & \beta \neq p+\alpha-s .\end{cases}
\end{aligned}
$$

Furthermore, $\left\langle P\left[\xi^{p} \bar{\xi}^{s} \varphi z^{\alpha}\right], \bar{z}^{\beta}\right\rangle=0=\left\langle z^{p+\alpha-s}, \bar{z}^{\beta}\right\rangle$ hold for all $\beta>0$. So we obtain

$$
\begin{aligned}
& P\left[\xi^{p} \bar{\xi}^{s} \varphi z^{\alpha}\right] \\
& =\left\{\begin{array}{cc}
\frac{2(p+\alpha) !(n+|p|+|\alpha|-|s|) !}{(p+\alpha-s) !(n-1+|\alpha|+|p|) !} & p+\alpha \geq s, \\
\times \widehat{\varphi}(2 n+2|\alpha|+|p|-|s|) z^{p+\alpha-s} & p+\alpha \geq s . \\
0 & p
\end{array}\right.
\end{aligned}
$$

Note that $\bar{\varphi}$ is still radial and $\overline{\widehat{\varphi}(n)}=\widehat{\bar{\varphi}}(n)$ for all $n \in N$, we have

$$
\begin{aligned}
& P\left[\bar{\xi}^{p} \xi^{s} \overline{\varphi z}^{\alpha}\right] \\
& =\left\{\begin{array}{cc}
\frac{2 s !(n+|s|-|\alpha|-|p|) !}{(s-\alpha-p) !(n-1+|s|) !} & p+\alpha \preceq s, \\
\times \overline{\widehat{\varphi}}(2 n+|s|-|p|) z^{s-\alpha-p} & p+\alpha \nless s . \\
0 & p
\end{array}\right.
\end{aligned}
$$

Thus

$$
\begin{aligned}
& T_{\xi^{p} \bar{\xi}_{\varphi}^{s}}\left(z^{\alpha}\right) \\
& =P\left[\xi^{p} \bar{\xi}^{s} \varphi z^{\alpha}\right]+\overline{P\left[\bar{\xi}^{p} \xi s \bar{\varphi} z^{\alpha}\right]}-P\left[\xi^{p} \bar{\xi}^{s} \varphi z^{\alpha}\right](0) \\
& =\left\{\begin{array}{cc}
\frac{2(p+\alpha) !(n+|p|+|\alpha|-|s|) !}{(p+\alpha-s) !(n-1+|\alpha|+|p|) !} & \\
\times \widehat{\varphi}(2 n+2|\alpha|+|p|-|s|) z^{p+\alpha-s} & p+\alpha \succeq s, \\
\frac{2 s !(n+|s|-|\alpha|-|p|) !}{(s-\alpha-p) !(n-1+|s|) !} & p+\alpha \preceq s, \\
\times \widehat{\varphi}(2 n+|s|-|p|) \bar{z}^{s-\alpha-p} & p+\alpha \ddagger s, \\
0 & p+\alpha \nsupseteq s .
\end{array}\right.
\end{aligned}
$$

Especially, if $p \perp s$, we know that $p+\alpha \geq s$ is equivalent to $\alpha \geq s$. Since $p, s$ are nonzero multi-indexes with $p \perp s$, there exists $i \in\{1,2, \ldots, n\}$ such that $p_{i} \neq 0, s_{i}=0$. This means that there doesn't exist multi-index $\alpha$ such that $p+\alpha \preceq s$. So

$$
\begin{aligned}
& T_{\xi \bar{p}^{s} \varphi}\left(z^{\alpha}\right) \\
& =\left\{\begin{array}{cc}
\frac{2(p+\alpha) !(n+|p|+|\alpha|-|s|) !}{(p+\alpha-s) !(n-1+|\alpha|+|p|) !} & \alpha \geq s, \\
\times \widehat{\varphi}(2 n+2|\alpha|+|p|-|s|) z^{p+\alpha-s} & \alpha \geq s .
\end{array}\right.
\end{aligned}
$$

Theorem 13. Let $p_{i}, s_{i}$ be multi-indexes and let $\varphi_{i}$ be integrable radial functions on $B_{n}$ such that $T_{\xi^{p_{i}} \bar{\xi}^{s_{i}} \varphi_{i}}$ is bounded $i=$ $1,2, \ldots, m$. If the product $T_{\xi^{p_{m}} \bar{\xi}^{s_{m}} \varphi_{m}} \cdots T_{\xi^{p_{1}} \bar{\xi}^{s_{1}} \varphi_{1}}$ is of finite rank, then $\varphi_{i}=0$ for some $i \in\{1,2, \ldots, m\}$.

Proof. We denote the product of Toeplitz operators $T_{\xi^{p m} \bar{\xi}^{s_{m}} \varphi_{m}} \cdots T_{\xi^{p_{1}} \bar{\xi}^{s_{1}} \varphi_{1}}$ by S. By Lemma 12, we have

$$
\begin{aligned}
& S\left(z^{\alpha}\right) \\
& =\prod_{i=m}^{2} T_{\xi^{p_{i}} \bar{\xi}^{s_{i}} \varphi_{i}}
\end{aligned}
$$

$$
\times \begin{cases}\frac{2\left(p_{1}+\alpha\right) !\left(n+\left|p_{1}\right|+|\alpha|-\left|s_{1}\right|\right) !}{\left(p_{1}+\alpha-s_{1}\right) !\left(n-1+|\alpha|-\left|p_{1}\right|\right) !} & \\ \quad \times \widehat{\varphi_{1}}\left(2 n+2|\alpha|+\left|p_{1}\right|-\left|s_{1}\right|\right) z^{p_{1}+\alpha-s_{1}} & p_{1}+\alpha \geq s_{1}, \\ \frac{2 s_{1} !\left(n+\left|s_{1}\right|-\left|p_{1}\right|-|\alpha|\right) !}{\left(s_{1}-p_{1}-\alpha\right) !\left(n-1+\left|s_{1}\right|\right) !} & \\ \times \widehat{\varphi_{1}}\left(2 n+\left|s_{1}\right|-\left|p_{1}\right|\right) \bar{z}^{s_{1}-\alpha-p_{1}} & p_{1}+\alpha \preceq s_{1}, \\ 0 & p_{1}+\alpha \geq s_{1}, \\ & p_{1}+\alpha \geq s_{1} .\end{cases}
$$


If $p_{1}+\alpha \succeq s_{1}, p_{2}+p_{1}+\alpha \succeq s_{1}+s_{2}, \ldots, p_{m}+\cdots+p_{1}+\alpha \succeq$ $s_{1}+\cdots+s_{m}$, we have

$$
\begin{aligned}
S\left(z^{\alpha}\right)= & 2^{m} a(\alpha) b(|\alpha|) \\
& \times \prod_{i=1}^{m} \widehat{\varphi}_{i}\left[2 n+2\left(|\alpha|+\sum_{j=0}^{i-1}\left(\left|p_{j}\right|-\left|s_{j}\right|\right)\right)+\left|p_{i}\right|-\left|s_{i}\right|\right] \\
& \times z^{\alpha+\sum_{i=1}^{m} p_{i}-s_{i}}
\end{aligned}
$$

where

$$
\begin{gathered}
a(\alpha)=\frac{\prod_{i=1}^{m}\left[\left(\alpha+\sum_{j=0}^{i-1}\left(p_{j}-s_{j}\right)+p_{i}\right) !\right]}{\prod_{i=1}^{m}\left[\left(\alpha+\sum_{j=1}^{i}\left(p_{j}-s_{j}\right)\right) !\right]}, \\
b(|\alpha|)=\frac{\prod_{i=1}^{m}\left[\left(n+|\alpha|+\sum_{j=1}^{i}\left(\left|p_{j}\right|-\left|s_{j}\right|\right)\right) !\right]}{\prod_{i=1}^{m}\left[\left(n-1+|\alpha|+\sum_{j=0}^{i-1}\left(\left|p_{j}\right|-\left|s_{j}\right|\right)+\left|p_{i}\right|\right) !\right]},
\end{gathered}
$$

$p_{0}=0$ and $s_{0}=0$.

Similarly, we have

$$
\begin{aligned}
& S\left(\overline{\mathrm{z}}^{\alpha}\right) \\
& =\prod_{i=m}^{2} T_{\xi^{p} \bar{\xi}^{s_{i}} \varphi_{i}} \\
& \\
& \times \begin{cases}\frac{2\left(s_{1}+\alpha\right) !\left(n+\left|s_{1}\right|+|\alpha|-\left|p_{1}\right|\right) !}{\left(s_{1}+\alpha-p_{1}\right) !\left(n-1+|\alpha|-\left|s_{1}\right|\right) !} & \\
\quad \times \widehat{\varphi_{1}}\left(2 n+2|\alpha|+\left|s_{1}\right|-\left|p_{1}\right|\right) z^{s_{1}+\alpha-p_{1}} & s_{1}+\alpha \geq p_{1}, \\
\frac{2 p_{1} !\left(n+\left|p_{1}\right|-\left|s_{1}\right|-|\alpha|\right) !}{\left(p_{1}-s_{1}-\alpha\right) !\left(n-1+\left|p_{1}\right|\right) !} & \\
\times \widehat{\varphi_{1}}\left(2 n+\left|p_{1}\right|-\left|s_{1}\right|\right) \bar{z}^{p_{1}-\alpha-s_{1}} & s_{1}+\alpha \preceq p_{1}, \\
0 & s_{1}+\alpha \geq p_{1}, \\
& s_{1}+\alpha \geq p_{1} .\end{cases}
\end{aligned}
$$

Then for $s_{1}+\alpha \geq p_{1}, s_{2}+s_{1}+\alpha \geq p_{1}+p_{2}, \ldots s_{m}+\cdots+s_{1}+\alpha \succeq$ $p_{1}+\cdots+p_{m}$, we have

$$
\begin{aligned}
S\left(\bar{z}^{\alpha}\right)= & 2^{m} a^{\prime}(\alpha) b^{\prime}(|\alpha|) \\
& \times \prod_{i=1}^{m} \widehat{\varphi}_{i}\left[2 n+2\left(|\alpha|+\sum_{j=0}^{i-1}\left(\left|s_{j}\right|-\left|p_{j}\right|\right)\right)+\left|s_{i}\right|-\left|p_{i}\right|\right] \\
& \times z^{\alpha+\sum_{i=1}^{m} s_{i}-p_{i}},
\end{aligned}
$$

where

$$
\begin{gathered}
a^{\prime}(\alpha)=\frac{\prod_{i=1}^{m}\left[\left(\alpha+\sum_{j=0}^{i-1}\left(s_{j}-p_{j}\right)+s_{i}\right) !\right]}{\prod_{i=1}^{m}\left[\left(\alpha+\sum_{j=1}^{i}\left(s_{j}-p_{j}\right)\right) !\right]}, \\
b^{\prime}(|\alpha|)=\frac{\prod_{i=1}^{m}\left[\left(n+|\alpha|+\sum_{j=1}^{i} \sum\left(\left|s_{j}\right|-\left|p_{j}\right|\right)\right) !\right]}{\prod_{i=1}^{m}\left[\left(n-1+|\alpha|+\sum_{j=0}^{i-1}\left(\left|s_{j}\right|-\left|p_{j}\right|\right)+\left|s_{i}\right|\right) !\right]} .
\end{gathered}
$$

Since $S$ has finite rank, we know that $\left\{S\left(z^{\alpha}\right)\right\}_{\alpha \geq 0}$ and $\left\{S\left(\bar{z}^{\alpha}\right)\right\}_{\alpha>0}$ must have finite rank. Then, there exists a multiindex $\alpha_{0}$ such that $p_{1}+\alpha_{0} \geq s_{1}, p_{2}+p_{1}+\alpha_{0} \geq s_{1}+s_{2}, \ldots, p_{m}+$ $\cdots+p_{1}+\alpha_{0} \succeq s_{1}+\cdots+s_{m}$ and

$$
S\left(z^{\alpha}\right)=0 \quad \forall \alpha \geq \alpha_{0}
$$

which is equivalent to

$$
\begin{gathered}
\widehat{\varphi_{m}}\left[2 n+2|\alpha|+2 \sum_{j=0}^{m-1}\left(\left|p_{j}\right|-\left|s_{j}\right|\right)+\left|p_{m}\right|-\left|s_{m}\right|\right] \ldots \\
\widehat{\varphi_{1}}\left[2 n+2|\alpha|+\left|p_{1}\right|-\left|s_{1}\right|\right]=0,
\end{gathered}
$$

for all $\alpha \geq \alpha_{0}$. In fact, we have $\alpha_{0}=\left(t_{1}, \ldots, t_{n}\right)$, in which $t_{j}=\max \left\{k_{1}^{(j)}, k_{2}^{(j)}, \ldots, k_{m}^{(j)}\right\}$ and $k_{i}^{j}=\max \left\{\left(s_{1}^{(j)}-p_{1}^{(j)}+s_{2}^{(j)}-\right.\right.$ $\left.\left.p_{2}^{(j)}+\cdots+s_{i}^{(j)}-p_{i}^{(j)}\right), 0\right\}, s_{i}=\left(s_{i}^{(1)}, \ldots, s_{i}^{(n)}\right), p_{i}=\left(p_{i}^{(1)}, \ldots, p_{i}^{(n)}\right)$, $i \in\{1,2, \ldots, m\}, j \in\{1,2, \ldots, n\}$.

Let $l=\min \left\{\left|p_{1}\right|-\left|s_{1}\right|, 2\left(\left|p_{1}\right|-\left|s_{1}\right|\right)+\left|p_{2}\right|-\right.$ $\left.\left|s_{2}\right|, \ldots, 2 \sum_{j=1}^{m-1}\left(\left|p_{j}\right|-\left|s_{j}\right|\right)+\left|p_{m}\right|-\left|s_{m}\right|\right\}$, we know that $2|\alpha|+l \geq$ 0 , for all $\alpha \geq \alpha_{0}$. Then (50) implies that

$$
\begin{gathered}
\left(r^{2\left(\sum_{j-1}^{m-1}\left(\left|p_{j}\right|-\left|s_{j}\right|\right)\right)+\left|p_{m}\right|-\left|s_{m}\right|-l} \varphi_{m}\right)^{\wedge}(2 n+2|\alpha|+l) \cdots \\
\left(r^{\left|p_{1}\right|-\left|s_{1}\right|-l} \varphi_{1}\right)^{\wedge}(2 n+2|\alpha|+l)=0,
\end{gathered}
$$

for all $\alpha \geq \alpha_{0}$. Since $\{2 n+2|\alpha|+l\}_{\alpha \succeq \alpha}$ is an arithmetic sequence, by Theorem 1, we obtain that there exists some $i$ such that $\varphi_{i}=0$.

\section{Finite Rank Commutators}

In this section, let $\varphi, \psi$ be two integrable radial functions on $B_{n}$. We now pass to investigate the commutator $\left[T_{\psi}, T_{\xi^{p} \bar{\xi}^{s} \varphi}\right],\left[T_{\xi^{p} \varphi}, T_{\xi^{s} \psi}\right]$ and $\left[T_{\xi^{p} \varphi}, T_{\bar{\xi}^{s} \psi}\right]$.

Theorem 14. Let $p$, s be two multi-indexes and let $\varphi$ and $\psi$ be two integrable radial functions on $B_{n}$ such that $T_{\psi}$ and $T_{\xi^{p} \bar{\xi}^{s} \varphi}$ are bounded operators. If $\psi$ is nonconstant and $\left[T_{\psi}, T_{\xi^{p} \bar{\xi}_{\varphi}^{s}}\right]$ has finite rank, then its rank is equal to zero.

Proof. Let $S$ denote the commutator $\left[T_{\psi}, T_{\xi^{p} \bar{\xi}_{\varphi}^{s}}\right]$. Since $S$ has finite rank on $L_{h}^{2}\left(B_{n}\right)$, we know that $\left\{S\left(z^{\alpha}\right)\right\}_{\alpha \succeq 0}$ and $\left\{S\left(\bar{z}^{\alpha}\right)\right\}_{\alpha>0}$ must have finite rank. 
By Lemma 12, we have if $p+\alpha \geq s$,

$$
\begin{aligned}
S\left(z^{\alpha}\right)= & \frac{2(p+\alpha) !(n+|p|+|\alpha|-|s|) !}{(p+\alpha-s) !(n-1+|p|+|\alpha|) !} \\
& \times \widehat{\varphi}(2 n+2|\alpha|+|p|-|s|) \\
& \times[2(n+|p|+|\alpha|-|s|) \widehat{\psi}(2 n+2|p|+2|\alpha|-2|s|) \\
& -2(n+|\alpha|) \widehat{\psi}(2 n+2|\alpha|)] z^{p+\alpha-s} .
\end{aligned}
$$

If $p+\alpha \preceq s$,

$$
\begin{aligned}
S\left(z^{\alpha}\right)= & \frac{2 s !(n+|s|-|\alpha|-|p|) !}{(s-\alpha-p) !(n-1+|s|) !} \widehat{\varphi}(2 n+|s|-|p|) \\
& \times[2(n+|s|-|\alpha|-|p|) \widehat{\psi}(2 n+2|s|-2|\alpha|-2|p|) \\
& -2(n+|\alpha|) \widehat{\psi}(2 n+2|\alpha|)] \bar{z}^{s-\alpha-p},
\end{aligned}
$$

if $p+\alpha \nsupseteq s, p+\alpha \not s$, we have $S\left(z^{\alpha}\right)=0$.

Since $\left\{S\left(z^{\alpha}\right)\right\}_{\alpha \succeq 0}$ has finite rank, then there must exist $\alpha_{0}$, such that $p+\alpha_{0} \geq s$ and $S\left(z^{\alpha}\right)=0$ for all $\alpha \geq \alpha_{0}$.

Similar to the proof of Theorem 4.4 in [7], we can imply $\varphi=0$ or $|p|=|s|$. It follows from (52) and (53) that

$$
S\left(z^{\alpha}\right)=0 \quad \forall \alpha \geq 0 .
$$

Similar to the discussions for $S\left(z^{\alpha}\right)$, we can imply that $S\left(\bar{z}^{\alpha}\right)=0$ for all $\alpha \geq 0$. In conclusion, the rank of $S$ is equal to zero.

Theorem 15. Let $p, s$ be two nonzero multi-indexes, let $\varphi$ and $\psi$ be two integrable radial functions on $B_{n}$, such that $T_{\xi^{p} \varphi}$ and $T_{\xi^{s} \psi}$ are bounded operators. If $\left[T_{\xi^{p} \varphi}, T_{\xi^{s} \psi}\right]$ has finite rank, then the rank of it is less than or equal to the number of $\alpha$ such that $\alpha \preceq p+s$, and $\left\{\left[T_{\xi^{p} \varphi}, T_{\xi^{s} \psi}\right]\left(z^{\alpha}\right)\right\}_{\alpha \succeq 0} \bigcup\left\{\left[T_{\xi^{p} \varphi}, T_{\xi^{s} \psi}\right]\left(\bar{z}^{\alpha}\right)\right\}_{\alpha>0} \subset$ $\bigvee_{\alpha \leq p+s}\left\{z^{p+s-\alpha}\right\}$, where $\bigvee_{\alpha \preceq s+p}\left\{z^{s+p-\alpha}\right\}=\operatorname{span}\left\{z^{s+p-\alpha}: \alpha \preceq\right.$ $s+p\}$.

Proof. Let $S$ denote the commutator $\left[T_{\xi^{p} \varphi}, T_{\xi^{s} \psi}\right]$. By Lemma 12, we have

$$
\begin{aligned}
S\left(z^{\alpha}\right)=2(n+ & |\alpha|+|p|+|s|) \\
\times[2 & (n+|\alpha|+|s|) \widehat{\psi}(2 n+2|\alpha|+|s|) \\
& \times \widehat{\varphi}(2 n+2|\alpha|+|p|+2|s|)-2(n+|\alpha|+|p|) \\
& \times \widehat{\psi}(2 n+2|\alpha|+2|p|+|s|) \\
& \times \widehat{\varphi}(2 n+2|\alpha|+|p|)] z^{p+\alpha+s} .
\end{aligned}
$$
that

If $\left\{S\left(z^{\alpha}\right)\right\}_{\alpha \succeq 0}$ has finite rank, there must exist $k \geq 0$ such

$$
S\left(z^{\alpha}\right)=0, \quad \forall|\alpha| \geq k
$$

Then, we deduce that

$$
\begin{aligned}
\widehat{1}(2 n+ & 2|p|+2|\alpha|) \widehat{\varphi}(2 n+2|s|+2|\alpha|+|p|) \\
& \times \widehat{\psi}(2 n+|s|+2|\alpha|) \\
= & \widehat{1}(2 n+2|s|+2|\alpha|) \widehat{\varphi}(2 n+2|\alpha|+|p|) \\
& \times \widehat{\psi}(2 n+|s|+2|\alpha|+2|p|), \quad|\alpha| \geq k .
\end{aligned}
$$

It follows from Remark 2 that

$$
\begin{aligned}
& \widehat{r^{2|p|} 1}(z) \sqrt{r^{2|s|+|p|}} \varphi(z) \widehat{r^{|s|} \psi}(z) \\
& =\widehat{r^{2|s|}} 1(z) \widehat{r|p|} \varphi(z) \widehat{r^{2|p|+|s|}} \psi(z),
\end{aligned}
$$

for all $\operatorname{Re} z>2 k$. Set $z=2 n+2|\alpha|$, we get that (57) holds for all $|\alpha| \geq 0$. Hence for all $\alpha \geq 0$, we have $S\left(z^{\alpha}\right)=0$. Therefore, the rank of $\left\{S\left(z^{\alpha}\right)\right\}_{\alpha \succ 0}$ is equal to zero.

For $S\left(\bar{z}^{\alpha}\right)$, we have if $\alpha \geq p+s$,

$$
\begin{aligned}
S\left(\bar{z}^{\alpha}\right)=\frac{4 \alpha !(n+|\alpha|-|p|-|s|) !}{(\alpha-p-s) !(n-1+|\alpha|) !} \\
\quad \times\left[\frac{(n+|\alpha|-|s|) !}{(n-1+|\alpha|-|s|) !} \widehat{\psi}(2 n+2|\alpha|-|s|)\right. \\
\quad \times \widehat{\varphi}(2 n+2|\alpha|-2|s|-|p|) \\
\quad-\frac{(n+|\alpha|-|p|) !}{(n-1+|\alpha|-|p|) !} \\
\quad \times \widehat{\psi}(2 n+2|\alpha|-2|p|-|s|) \\
\quad \times \widehat{\varphi}(2 n+2|\alpha|-|p|)] \bar{z}^{\alpha-p-s} .
\end{aligned}
$$

If $\alpha \nsupseteq p, \alpha \not \leq p, \alpha \nsupseteq s, \alpha \not s$, we have $S\left(\bar{z}^{\alpha}\right)=0$, if $\alpha \geq p$ but $\alpha \nsupseteq p+s, \alpha \not p+s$, we have $S\left(\bar{z}^{\alpha}\right)=0$, if $\alpha \geq s$ but $\alpha \nsupseteq p+s, \alpha \nsubseteq p+s$, then $S\left(\bar{z}^{\alpha}\right)=0$.

If $s \preceq \alpha \preceq p+s$ or $p \preceq \alpha \preceq p+s$ or $\alpha \preceq p$ or $\alpha \preceq s$,

$$
S\left(\bar{z}^{\alpha}\right)=C(\alpha) z^{p+s-\alpha}
$$

where $C(\alpha)$ is a constant that depends on $\alpha$.

If $\left\{S\left(\bar{z}^{\alpha}\right)\right\}_{\alpha \succ 0}$ has finite rank, use the same method as above, we have

$$
S\left(\bar{z}^{\alpha}\right)=0, \quad \forall \alpha \geq p+s
$$

this means $\left\{S\left(\bar{z}^{\alpha}\right)\right\}_{\alpha \succeq 0} \subset \bigvee_{\alpha \leq p+s}\left\{z^{p+s-\alpha}\right\}$, and the rank of $S\left(\bar{z}^{\alpha}\right)$ is at most equal to the number of $\alpha$ such that $\alpha \in G$. In which $G=\{\alpha: s \preceq \alpha \preceq p+s\} \bigcup\{\alpha: p \preceq \alpha \preceq p+s\} \bigcup\{\alpha$ : $\alpha \preceq p\} \bigcup\{\alpha: \alpha \preceq s\}$ and $G \subseteq\{\alpha \preceq p+s\}$. The proof is complete.

Example 16. In the case of $n=2$, we give an example to Theorem 15.

From the proof of Theorem 15 we know that

$$
T_{\xi^{p} r^{m}} T_{\xi^{s} f}\left(z^{\alpha}\right)=T_{\xi^{s} f} T_{\xi^{p} r^{m}}\left(z^{\alpha}\right), \quad \forall \alpha \succeq 0 .
$$


Below we will construct a radial function $f$, such that

$$
T_{\xi^{p} r^{m}} T_{\xi^{s} f}\left(\bar{z}^{\alpha}\right)=T_{\xi^{s} f} T_{\xi^{p} r^{m}}\left(\bar{z}^{\alpha}\right), \quad \forall \alpha \geq p+s,
$$

where $p, s$ are multi-indexes, $\xi \in S_{2}$ and $m \geq 0$.

Equation (63) implies that for $\alpha \geq p+s$,

$$
\begin{aligned}
& \frac{r^{-\widehat{|s|-2|p|}} f(2|\alpha|+4+2|p|)}{r^{-\widehat{s|-2| p \mid}} f(2|\alpha|+4)} \\
& \quad=\frac{(2|\alpha|+4-2|p|)(2|\alpha|+4-|p|+m-2|s|)}{(2|\alpha|+4-2|s|)(2|\alpha|+4-|p|+m)} .
\end{aligned}
$$

Now, using Remark 2, we obtain that

$$
\frac{r^{\widehat{|s|-2|p|}} f(z+2|p|)}{r^{-\widehat{|s|-2|p|}} f(z)}=\frac{(z-2|p|)(z-|p|+m-2|s|)}{(z-2|s|)(z-|p|+m)},
$$

for all $\operatorname{Re} z \geq 2|p|+2|s|+4$. by

Let $F$ be the analytic function defined for $\operatorname{Re} z \geq 2|p|+2|s|$

$$
F(z)=\frac{\Gamma((z-2|p|) / 2|p|) \Gamma((z-|p|-2|s|+m) / 2|p|)}{\Gamma((z-2|s|) / 2|p|) \Gamma((z-|p|+m) / 2|p|)},
$$

where $\Gamma$ denote the gamma function. Then using the wellknown identity $\Gamma(z+1)=z \Gamma(z)$, (65) implies that

$$
\begin{array}{r}
\frac{r^{\widehat{|s|-2|p|}} f(z+2|p|)}{r^{-\widehat{|s|-2|p|}} f(z)}=\frac{F(z+2|p|)}{F(z)}, \\
\text { for } \operatorname{Re} z>2|p|+2|s| .
\end{array}
$$

Equation (67) combined with [30, Lemma 6, page 1468] gives us that there exists a constant $c$ such that

$$
r^{\widehat{|s|-2|p|}} f(z)=c F(z) \text { for } \operatorname{Re} z>2|p|+2|s| \text {. }
$$

For a choice of $p=(2,0), s=(0,1)$, and $m=6$, again using the identity $\Gamma(z+1)=z \Gamma(z)$, one can see that

$$
F(z)=\frac{4(z-2)}{z(z-4)}=2\left[\frac{1}{z}+\frac{1}{z-4}\right] .
$$

Since $\widehat{1}(z)=1 / z, \widehat{r^{-4}}(z)=1 /(z-4)$, then (68) becomes

$$
\widehat{r^{-5} f}(z)=c\left[\widehat{1}(z)+\widehat{r^{-4}}(z)\right], \quad \text { for } \operatorname{Re} z>6
$$

Now the proceeding equation and Remark 2 imply that

$$
f(r)=c\left(r^{5}+r\right), \quad \text { where } c \text { is a constant. }
$$

It is clear that the function $f$ is bounded, so Toeplitz operator $T_{\xi^{(0,1)} f}$ is bounded.
Finally, by taking the constant $c$ to be equal to 1 , the radial function $f(r)=r^{5}+r$ satisfies

$$
\begin{gathered}
T_{\xi^{(2,0)} r^{6}} T_{\xi^{(0,1)}\left(r^{5}+r\right)}\left(z^{\alpha}\right)=T_{\xi^{(0,1)}\left(r^{5}+r\right)} T_{\xi^{(2,0)} r^{6}}\left(z^{\alpha}\right), \quad \forall \alpha \geq 0, \\
T_{\xi^{(2,0)} r^{6}} T_{\xi^{(0,1)}\left(r^{5}+r\right)}\left(\bar{z}^{\alpha}\right)=T_{\xi^{(0,1)}\left(r^{5}+r\right)} T_{\xi^{(2,0)} r^{6}}\left(\bar{z}^{\alpha}\right), \quad \forall \alpha \geq(2,1) .
\end{gathered}
$$

For $\alpha=(2,1), \alpha_{1}=(1,1), \alpha_{2}=(0,1), \alpha_{3}=(2,0), \alpha_{4}=$ $(1,0)$, we know that $\alpha_{i} \preceq \alpha,(i=1,2,3,4)$. However, using Lemma 12 , it is easy to see that

$$
\begin{gathered}
T_{\xi^{(2,0)} r^{6}} T_{\xi^{(0,1)}\left(r^{5}+r\right)}\left(\bar{z}^{(1,1)}\right)=\frac{5}{36} z^{(1,0)}, \\
T_{\xi^{(0,1)}\left(r^{5}+r\right)} T_{\xi^{(2,0)} r^{6}}\left(\bar{z}^{(1,1)}\right)=0, \\
T_{\xi^{(2,0)} r^{6}} T_{\xi^{(0,1)}\left(r^{5}+r\right)}\left(\bar{z}^{(0,1)}\right)=\frac{16}{45} z^{(2,0)}, \\
T_{\xi^{(0,1)}\left(r^{5}+r\right)} T_{\xi^{(2,0)} r^{6}}\left(\bar{z}^{(0,1)}\right)=0, \\
T_{\xi^{(2,0)} r^{6}} T_{\xi^{(0,1)}\left(r^{5}+r\right)}\left(\bar{z}^{(2,0)}\right)=0, \\
T_{\xi^{(0,1)}\left(r^{5}+r\right)} T_{\xi^{(2,0)} r^{6}}\left(\bar{z}^{(2,0)}\right)=\frac{8}{45} z^{(0,1)}, \\
T_{\xi^{(2,0)} r^{6}} T_{\xi^{(0,1)}\left(r^{5}+r\right)}\left(\bar{z}^{(1,0)}\right)=0, \\
T_{\xi^{(0,1)}\left(r^{5}+r\right)} T_{\xi^{(2,0)} r^{6}}\left(\bar{z}^{(1,0)}\right)=\frac{5}{9} z^{(1,1)} .
\end{gathered}
$$

Therefore the commutator $\left[T_{\xi^{(2,0)} r^{6}}, T_{\xi^{(0,1)}\left(r^{5}+r\right)}\right]$ has rank four.

Theorem 17. Let $p$, s be two nonzero multi-indexes with $p \perp$ $s$, let $\varphi$ and $\psi$ be two integrable radial functions on $B_{n}$, such that $T_{\xi^{p} \varphi}$ and $T_{\bar{\xi}^{s} \psi}$ are bounded operators. If $\left[T_{\xi^{p} \varphi}, T_{\bar{\xi}^{s} \psi}\right]$ has finite rank, then the rank of it is equal to zero.

Proof. Let $S$ denote the commutator $\left[T_{\xi^{p} \varphi}, T_{\bar{\xi}_{\psi}^{s}}\right]$. Since $p \perp s$, it is easy to see that $p+\alpha \geq s$ is equivalent to $\alpha \geq s$ for all multi-index $\alpha$, and there does not exist multi-index $\alpha^{\prime}$ such that $p+\alpha^{\prime} \preceq s$. Furthermore, it is impossible that there exists $\alpha_{0}$ such that $\alpha_{0} \preceq s$ and $s \preceq \alpha_{0}+p$.

If $\left\{S\left(z^{\alpha}\right)\right\}_{\alpha \succeq 0}$ has finite rank, there exists $\alpha_{0} \geq 0$ such that

$$
S\left(z^{\alpha}\right)=0, \quad \forall \alpha \geq s+\alpha_{0} .
$$

Since $p \perp s$, we have $\alpha !(p+\alpha-s) !=(\alpha+p) !(\alpha-s)$ !. So

$$
\begin{aligned}
S\left(z^{\alpha}\right)=\frac{\alpha !}{(\alpha-s) !}[ & \frac{(n+|\alpha|-|s|) !}{(n-1+|\alpha|) !} 2(n+|\alpha|+|p|-|s|) \\
& \times \widehat{\psi}(2 n+2|\alpha|-|s|) \\
& \times \widehat{\varphi}(2 n+2|\alpha|-2|s|+|p|)
\end{aligned}
$$




$$
\begin{aligned}
& -\frac{(n+|\alpha|+|p|-|s|) !}{(n-1+|p|+|\alpha|) !} 2(n+|\alpha|+|p|) \\
& \times \widehat{\psi}(2 n+2|\alpha|+2|p|-|s|) \\
& \times \widehat{\varphi}(2 n+2|\alpha|+|p|)] z^{p+\alpha-s}
\end{aligned}
$$

As in the proof of Theorem 15, we have $S\left(z^{\alpha}\right)=0$ for all $\alpha \geq s$, this means that the rank of $\left\{S\left(z^{\alpha}\right)\right\}_{\alpha \geq 0}$ is equal to zero.

Similarly, we can prove that the rank of $\left\{S\left(\bar{z}^{\alpha}\right)\right\}_{\alpha>0}$ is equal to zero.

\section{Finite Rank Semicommutators}

We will discuss the semicommutators of two Toeplitz operators with quasihomogeneous symbols, the results in this section are different from those in $[9,14]$.

Theorem 18. Let $p, s$ be two multi-indexes and let $\varphi$ and $\psi$ be two integrable radial functions on $B_{n}$, such that $T_{\psi}, T_{\xi^{p} \bar{\xi}^{s} \varphi}$ are bounded operators.

(a) If $s \geq p,\left(T_{\psi}, T_{\xi^{p} \bar{\xi}_{\varphi}^{s}}\right]$ has finite rank, then its rank is at most equal to the number of $\alpha$ such that $\alpha \preceq s-$ $p$ and $\left\{\left(T_{\psi}, T_{\xi^{p} \bar{\xi}_{\varphi}^{s}}\right]\left(z^{\alpha}\right)\right\}_{\alpha \succeq 0} \bigcup\left\{\left(T_{\psi}, T_{\xi^{p} \bar{\xi}_{\varphi}^{s}}\right]\left(\bar{z}^{\alpha}\right)\right\}_{\alpha \succ 0} \subseteq$ $\bigvee_{\alpha \preceq s-p}\left\{\bar{z}^{s-p-\alpha}\right\}$.

(b) If $s \preceq p,\left(T_{\psi}, T_{\xi^{p} \bar{\xi}_{\varphi}^{s}}\right]$ has finite rank, then its rank is at most equal to the number of $\alpha$ such that $\alpha \preceq p-$ $s$ and $\left\{\left(T_{\psi}, T_{\xi^{p} \bar{\xi}^{s} \varphi}\right]\left(z^{\alpha}\right)\right\}_{\alpha \succeq 0} \bigcup\left\{\left(T_{\psi}, T_{\xi^{p} \bar{\xi}^{s} \varphi}\right]\left(\bar{z}^{\alpha}\right)\right\}_{\alpha \succ 0} \subseteq$ $\bigvee_{\alpha \leq p-s}\left\{z^{p-s-\alpha}\right\}$.

(c) If $s \nsupseteq p, s \nless p$, and $\left(T_{\psi}, T_{\xi^{p} \bar{\xi}_{\varphi}^{s}}\right]$ has finite rank, then its rank is equal to zero.

Proof. Let $S$ denote the semicommutator $\left(T_{\psi}, T_{\xi^{p} \bar{\xi}_{\varphi}^{s}}\right]$.

If $s+\alpha \geq p$, we compute that

$$
\begin{aligned}
S\left(\bar{z}^{\alpha}\right)= & \frac{2(s+\alpha) !(n+|s|+|\alpha|-|p|) !}{(s+\alpha-p) !(n-1+|s|+|\alpha|) !} \\
& \times[\widehat{\varphi \psi}(2 n+2|\alpha|+|s|-|p|) \\
& \quad-2(n+|s|+|\alpha|-|p|) \\
& \times \widehat{\psi}(2 n+2|s|+2|\alpha|-2|p|) \\
& \times \widehat{\varphi}(2 n+2|\alpha|+|s|-|p|)] \bar{z}^{s+\alpha-p} .
\end{aligned}
$$

$$
\begin{aligned}
& \text { If } s+\alpha \preceq p, \\
& \qquad \begin{aligned}
S\left(\bar{z}^{\alpha}\right)= & \frac{2 p !(n+|p|-|\alpha|-|s|) !}{(p-\alpha-s) !(n-1+|p|) !} \\
& \times[\widehat{\varphi \psi}(2 n+|p|-|s|) \\
& -2(n+|p|-|\alpha|-|s|) \\
& \times \widehat{\psi}(2 n+2|p|-2|\alpha|-2|s|) \\
& \times \widehat{\varphi}(2 n+|p|-|s|)] z^{p-\alpha-s},
\end{aligned}
\end{aligned}
$$

if $s+\alpha \nsupseteq p$ and $s+\alpha \not p$, then $S\left(\bar{z}^{\alpha}\right)=0$. Next we consider three cases.

Case $1(p \succeq s)$. If $\left\{S\left(\bar{z}^{\alpha}\right)\right\}_{\alpha>0}$ has finite rank, there exists $\alpha_{0} \succeq$ 0 such that

$$
S\left(\bar{z}^{\alpha}\right)=0 \quad \forall \alpha \geq p-s+\alpha_{0} .
$$

Similar to the proof of Theorem 15 , we deduce that $S\left(\bar{z}^{\alpha}\right)=0$ for all $\alpha \succeq p-s$. Therefore, the rank of $\left\{S\left(\bar{z}^{\alpha}\right)\right]_{\alpha>0}$ is at most equal to the number of $\alpha$ such that $\alpha \preceq p-s$, and $\left\{S\left(\bar{z}^{\alpha}\right)\right\}_{\alpha>0} \subseteq$ $\bigvee_{\alpha \preceq p-s}\left\{z^{p-s-\alpha}\right\}$.

Case $2(s \geq p)$. Since $s \geq p$, we know that $s+\alpha \succeq p$ for any multi-index $\alpha$. This means that (77) for all $\alpha \geq 0$. If $\left\{S\left(\bar{z}^{\alpha}\right)\right\}_{\alpha>0}$ has finite rank, again using the idea of proof of theorem, we conclude that the rank of $\left\{S\left(\bar{z}^{\alpha}\right)\right\}_{\alpha>0}$ is equal to zero.

Case $3(s \nsupseteq p, s \nless p)$. For $s=\left(s_{1}, \ldots, s_{n}\right), p=\left(p_{1}, \ldots, p_{n}\right)$, without loss of generality, we assume $s_{1}<p_{1}$ but $s_{i} \geq p_{i}(i \geq$ $2)$. Since $s_{1}<p_{1}$, hence $p_{1}+\delta>s_{1}$ for any nonnegative integer $\delta$. So there does not exist multi-index $\alpha$, such that $p+\alpha \preceq s$. But if $\alpha I=\left(0, s_{2}-p_{2}, \ldots, s_{n}-p_{n}\right)$, it is clear that $p+\alpha^{\prime} \geq s$. So for any $s \nsupseteq p$ and $s \nless \neq p$, there exists $\alpha_{0}=\left(\max \left\{\left(s_{1}-\right.\right.\right.$ $\left.\left.\left.p_{1}\right), 0\right\}, \ldots, \max \left\{\left(s_{n}-p_{n}\right), 0\right\}\right)$, such that $p+\alpha_{0} \geq s$, but there does not exist $\alpha^{\prime}$ such that $p+\alpha^{\prime} \preceq s$. Hence, we have (77) holding for $\alpha \geq \alpha_{0}$, and for $\alpha \geq \alpha_{0} S\left(\bar{z}^{\alpha}\right)=0$.

If $\left\{S\left(\bar{z}^{\alpha}\right)\right\}_{\alpha \succ 0}$ has finite rank, there exists $\alpha^{\prime} \geq 0$ such that

$$
S\left(\bar{z}^{\alpha}\right)=0, \quad \forall \alpha \geq \alpha_{0}+\alpha^{\prime} .
$$

As in the proof of Case 1, we can imply that

$$
S\left(\bar{z}^{\alpha}\right)=0, \quad \forall \alpha \succeq \alpha_{0} .
$$

This means that the rank of $\left\{S\left(\bar{z}^{\alpha}\right)\right\}_{\alpha>0}$ is equal to zero.

Similar to the discussions for $S\left(\bar{z}^{\alpha}\right)$, we obtain that

(1) if $s \geq p$, the rank of $S\left(z^{\alpha}\right)$ is at most equal to the number of $\alpha$ such that $\alpha \preceq s-p$, and $\left\{S\left(z^{\alpha}\right)\right\}_{\alpha \succeq 0} \subseteq$ $\bigvee_{\alpha \leq s-p}\left\{\bar{z}^{s-p-\alpha}\right\}$,

(2) if $s \preceq p$, the rank of $S\left(z^{\alpha}\right)$ is equal to zero,

(3) if $s \geq p$ and $s \not \subset p$, then the rank of $S\left(z^{\alpha}\right)$ is equal to zero. The proof is completed.

Remark 19. (1) If $p, s$ are two nonzero multi-indexes with $p \perp$ $s$, and if $\left(T_{\psi}, T_{\xi p \bar{\xi}_{\varphi}^{s}}\right]$ has finite rank, then the rank of it is equal to zero. 
(2) If $p=0,\left(T_{\psi}, T_{\bar{\xi}^{s} \varphi}\right]$ has finite rank, then the rank of it is at most equal to the number of $\alpha$ such that $\alpha \preceq s$ and $\left\{\left(T_{\psi}, T_{\bar{\xi}_{\varphi}^{s}}\right]\left(z^{\alpha}\right)\right\}_{\alpha \succeq 0} \bigcup\left\{\left(T_{\psi}, T_{\bar{\xi}_{\varphi}^{s}}\right]\left(\bar{z}^{\alpha}\right)\right\}_{\alpha \succ 0} \subseteq \bigvee_{\alpha \leq s}\left\{\bar{z}^{s-\alpha}\right\}$.

(3) If $\left(T_{\psi}, T_{\xi^{p} \varphi}\right]$ has finite rank, then the rank of it is at most equal to the number of $\alpha$ such that $\alpha \preceq p$ and $\left\{\left(T_{\psi}, T_{\xi^{p} \varphi}\right]\left(z^{\alpha}\right)\right\}_{\alpha \geq 0} \bigcup\left\{\left(T_{\psi}, T_{\xi^{p} \varphi}\right]\left(\bar{z}^{\alpha}\right)\right\}_{\alpha>0} \subseteq \bigvee_{\alpha \preceq p}\left\{z^{p-\alpha}\right\}$.

Example 20. In the case of $n=2$, we give an example to Theorem 18 (Remark 19). Similar to Example 16, there exist $\psi=r^{6}, \varphi=r^{-2}$ and $p=(1,1)$ such that

$$
\begin{gathered}
T_{r^{6}} T_{\xi^{(1,1)} r^{-2}}\left(z^{\alpha}\right)=T_{\xi^{(1,1)} r^{4}}\left(z^{\alpha}\right), \quad \forall \alpha \succeq 0, \\
T_{r^{6}} T_{\xi^{(1,1)} r^{-2}}\left(\bar{z}^{\alpha}\right)=T_{\xi^{(1,1)} r^{4}}\left(\bar{z}^{\alpha}\right), \quad \forall \alpha \geq(1,1) .
\end{gathered}
$$

Using Lemma 12, we have

$$
\begin{aligned}
T_{r^{6}} T_{\xi^{(1,1)} r^{-2}}\left(\bar{z}^{(1,0)}\right) & =\frac{1}{4} z^{(0,1)}, \\
T_{\xi^{(1,1)} r^{4}}\left(\bar{z}^{(1,0)}\right) & =\frac{1}{5} z^{(0,1)}, \\
T_{r^{6}} T_{\xi^{(1,1)} r^{-2}}\left(\bar{z}^{(0,1)}\right) & =\frac{1}{4} z^{(1,0)}, \\
T_{\xi^{(1,1)} r^{4}}\left(\bar{z}^{(0,1)}\right) & =\frac{1}{5} z^{(1,0)}
\end{aligned}
$$

so the rank of $\left(T_{r^{6}}, T_{\xi^{(1,1)} r^{-2}}\right]$ is equal to two.

Theorem 21. Let $p, s$ be two nonzero multi-indexes, let $\varphi$ and $\psi$ be two integrable radial functions, such that $T_{\xi^{p}}, T_{\xi^{s}} \psi$ are bounded operators. If $\left(T_{\xi^{p} \varphi}, T_{\xi^{s} \psi}\right]$ has finite rank, then its rank is at most equal to the number of $\alpha$ such that $\alpha \preceq$ $p+s$, and $\left\{\left(T_{\xi^{p} \varphi}, T_{\xi^{s} \psi}\right]\left(z^{\alpha}\right)\right\}_{\alpha \geq 0} \bigcup\left\{\left(T_{\xi^{p} \varphi}, T_{\xi^{s} \psi}\right]\left(\bar{z}^{\alpha}\right)\right\}_{\alpha>0} \subseteq$ $\bigvee_{\alpha \preceq p+s}\left\{z^{p+s-\alpha}\right\}$

Proof. The proof of this Theorem is similar to that of Theorem 15. Here, we omit it.

Example 22. In case of $n=2$, we give an example to Theorem 21. As we construct Example 16, let $\varphi=r^{2}, \psi=r^{6}$, and $p=(1,0), s=(1,1)$, we have

$$
\begin{gathered}
T_{\xi^{(1,0)} r^{2}} T_{\xi^{(1,1)} r^{6}}\left(z^{\alpha}\right)=T_{\xi^{(2,1)} r^{8}}\left(z^{\alpha}\right), \quad \forall \alpha \geq 0, \\
T_{\xi^{(1,0)} r^{2}} T_{\xi^{(1,1)} r^{6}}\left(\bar{z}^{\alpha}\right)=T_{\xi^{(2,1)} r^{8}}\left(\bar{z}^{\alpha}\right), \quad \forall \alpha \geq(2,1) .
\end{gathered}
$$

However,

$$
\begin{gathered}
T_{\xi^{(1,0)} r^{2}} T_{\xi^{(1,1)} r^{6}}\left(\bar{z}^{(2,0)}\right)=0, \\
T_{\xi^{(2,1)} r^{8}}\left(\bar{z}^{(2,0)}\right)=\frac{1}{15} z^{(0,1)}, \\
T_{\xi^{(1,0)} r^{2}} T_{\xi^{(1,1)} r^{6}}\left(\bar{z}^{(1,1)}\right)=\frac{1}{21} z^{(1,0)}, \\
T_{\xi^{(2,1)} r^{8}}\left(\bar{z}^{(1,1)}\right)=\frac{1}{15} z^{(1,0)}, \\
T_{\xi^{(1,0)} r^{2}} T_{\xi^{(1,1)} r^{6}}\left(\bar{z}^{(1,0)}\right)=\frac{4}{27} z^{(1,1)},
\end{gathered}
$$

$$
\begin{aligned}
& T_{\xi^{(2,1)} r^{8}}\left(\bar{z}^{(1,0)}\right)=\frac{4}{15} z^{(1,1)}, \\
& T_{\xi^{(1,0)} r^{2}} T_{\xi^{(1,1)} r^{6}}\left(\bar{z}^{(0,1)}\right)=\frac{4}{27} z^{(2,0)}, \\
& T_{\xi^{(2,1)} r^{8}}\left(\bar{z}^{(0,1)}\right)=\frac{2}{15} z^{(2,0)},
\end{aligned}
$$

Therefore the rank of $\left(T_{\xi^{(1,0)} r^{2}}, T_{\xi^{(1,1)} \mathrm{r}^{6}}\right]$ is equal to four.

Theorem 23. Let $p, s$ be two nonzero multi-indexes with $p \perp$ $s$, let $\varphi$ and $\psi$ be two integrable radial functions on $B_{n}$, such that $T_{\xi^{p} \varphi}$ and $T_{\bar{\xi}_{\psi}^{s}}$ are bounded operators. If $\left(T_{\xi^{p} \varphi}, T_{\bar{\xi}^{s} \psi}\right]$ has finite rank, then the rank of it is equal to zero.

Proof. The proof of this Theorem is similar to that of Theorem 17.

\section{Acknowledgments}

The authors would like to thank the referees for their excellent suggestions. This research is supported by NSFC (Grant nos. 10971020, and 11271059) and Research Fund for the Doctoral Program of Higher Education of China.

\section{References}

[1] A. Brown and P. R. Halmos, "Algebraic properties of Toeplitz operators," Journal für die Reine und Angewandte Mathematik, vol. 1964, no. 213, pp. 89-102, 1963.

[2] P. Ahern and Ž. Č. Čučković, "A theorem of Brown-Halmos type for Bergman space Toeplitz operators," Journal of Functional Analysis, vol. 187, no. 1, pp. 200-210, 2001.

[3] P. Ahern, "On the range of the Berezin transform," Journal of Functional Analysis, vol. 215, no. 1, pp. 206-216, 2004.

[4] I. Louhichi, E. Strouse, and L. Zakariasy, "Products of toeplitz operators on the bergman space," Integral Equations and Operator Theory, vol. 54, no. 4, pp. 525-539, 2006.

[5] X. Ding, "Products of Toeplitz operators on the polydisk," Integral Equations and Operator Theory, vol. 45, no. 4, pp. 389403, 2003.

[6] B. R. Choe, Y. J. Lee, K. Nam, and D. Zheng, "Products of Bergman space Toeplitz operators on the polydisk," Mathematische Annalen, vol. 337, no. 2, pp. 295-316, 2007.

[7] Z. H. Zhou and X. T. Dong, "Algebraic properties of toeplitz operators with radial symbols on the bergman space of the unit ball," Integral Equations and Operator Theory, vol. 64, no. 1, pp. 137-154, 2009.

[8] X. T. Dong and Z. H. Zhou, "Algebraic properties of toeplitz operators with separately quasihomogeneous symbols on the bergman space of the unit ball," Journal of Operator Theory, vol. 66, no. 1, pp. 193-207, 2011.

[9] Y. F. Lu and B. Zhang, "Finite rank commutator of Toeplitz operators with quasihomogeneous symbols on the unit ball," Acta Mathematica Sinic. In press.

[10] K. Y. Guo, "A problem on products of toeplitz operators," Proceedings of the American Mathematical Society, vol. 124, no. 3, pp. 869-871, 1996. 
[11] C. Gu, "Products of several Toeplitz operators," Journal of Functional Analysis, vol. 171, no. 2, pp. 483-527, 2000.

[12] A. Aleman and D. Vukotić, "Zero products of Toeplitz operators," Duke Mathematical Journal, vol. 148, no. 3, pp. 373-403, 2009.

[13] P. Ahern and Ž. Č. Čučković, "Some examples related to BrownHalmos theorem for the Bergman space," Acta Scientiarum Mathematicarum Szeged, vol. 70, pp. 373-378, 2004.

[14] Ž. Č. Čučković and I. Louhichi, "Finite rank commutators and semicommutators of quasihomogeneous Toeplitz operators," Complex Analysis and Operator Theory, vol. 2, no. 3, pp. 429439, 2008.

[15] B. Choe and H. Koo, "Zero products of Toeplitz operators with harmonic symbols," Journal of Functional Analysis, vol. 233, no. 2, pp. 307-334, 2006.

[16] B. R. Choe, H. Koo, and Y. J. Lee, "Zero products of toeplitz operators with n-harmonic symbols," Integral Equations and Operator Theory, vol. 57, no. 1, pp. 43-66, 2007.

[17] B. R. Choe, H. Koo, and Y. J. Lee, "Finite rank Toeplitz products with harmonic symbols," Journal of Mathematical Analysis and Applications, vol. 343, no. 1, pp. 81-98, 2008.

[18] T. Le, "Finite-rank products of toeplitz operators in several complex variables," Integral Equations and Operator Theory, vol. 63, no. 4, pp. 547-555, 2009.

[19] S. Axler, S.-Y. A. Chang, and D. Sarason, "Products of Toeplitz operators," Integral Equations and Operator Theory, vol. 1, no. 3, pp. 285-309, 1978.

[20] X. Ding and D. Zheng, "Finite rank commutator of toeplitz operators or hankel operators," Houston Journal of Mathematics, vol. 34, no. 4, pp. 1099-1119, 2008.

[21] K. Guo, S. Sun, and D. Zheng, "Finite rank commutators and semicommutators of Toeplitz operators with harmonic symbols," Illinois Journal of Mathematics, vol. 51, no. 2, pp. 583596, 2007.

[22] D. H. Luecking, "Finite rank toeplitz operators on the bergman space," Proceedings of the American Mathematical Society, vol. 136, no. 5, pp. 1717-1723, 2008.

[23] B. Zhang, Y. Y. Shi, and Y. F. Lu, "Algebraic properties of Toeplitz operators on the polydisk," Abstract and Applied Analysis, vol. 2011, Article ID 962313, 18 pages, 2011.

[24] B. R. Choe and Y. J. Lee, "Commuting toeplitz operators on the harmonic Bergman space," Michigan Mathematical Journal, vol. 46, no. 1, pp. 163-174, 1999.

[25] B. R. Choe and J. L. Young, "Commutants of analytic Toeplitz operators on the harmonic Bergman space," Integral Equations and Operator Theory, vol. 50, no. 4, pp. 559-564, 2004.

[26] Y. J. Lee and K. Zhu, "Some differential and integral equations with applications to Toeplitz operators," Integral Equations and Operator Theory, vol. 44, no. 4, pp. 466-479, 2002.

[27] B. R. Choe and K. S. Nam, "Note on commuting toeplitz operators on the pluriharmonic bergman space," Journal of the Korean Mathematical Society, vol. 43, no. 2, pp. 259-269, 2006.

[28] X. T. Dong and Z. H. Zhou, "Products of toeplitz operators on the harmonic bergman space," Proceedings of the American Mathematical Society, vol. 138, no. 5, pp. 1765-1773, 2010.

[29] R. Remmert, Classical Topics in Complex Function Theory, Graduate Texts in Methematics, vol. 172, Springer, New York, NY, USA, 1998.

[30] I. Louhichi, "Powers and roots of Toeplitz operators," Proceedings of the American Mathematical Society, vol. 135, no. 5, pp. 1465-1475, 2007. 


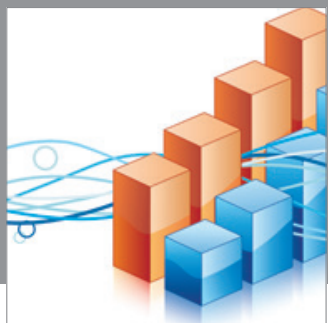

Advances in

Operations Research

mansans

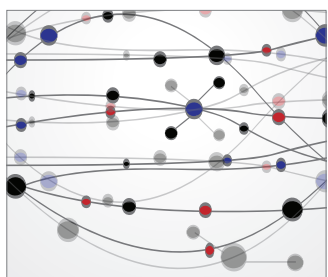

The Scientific World Journal
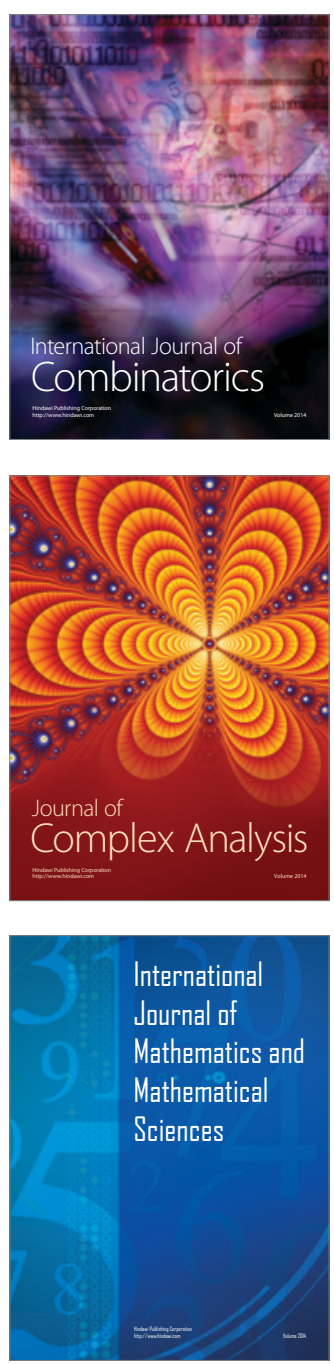
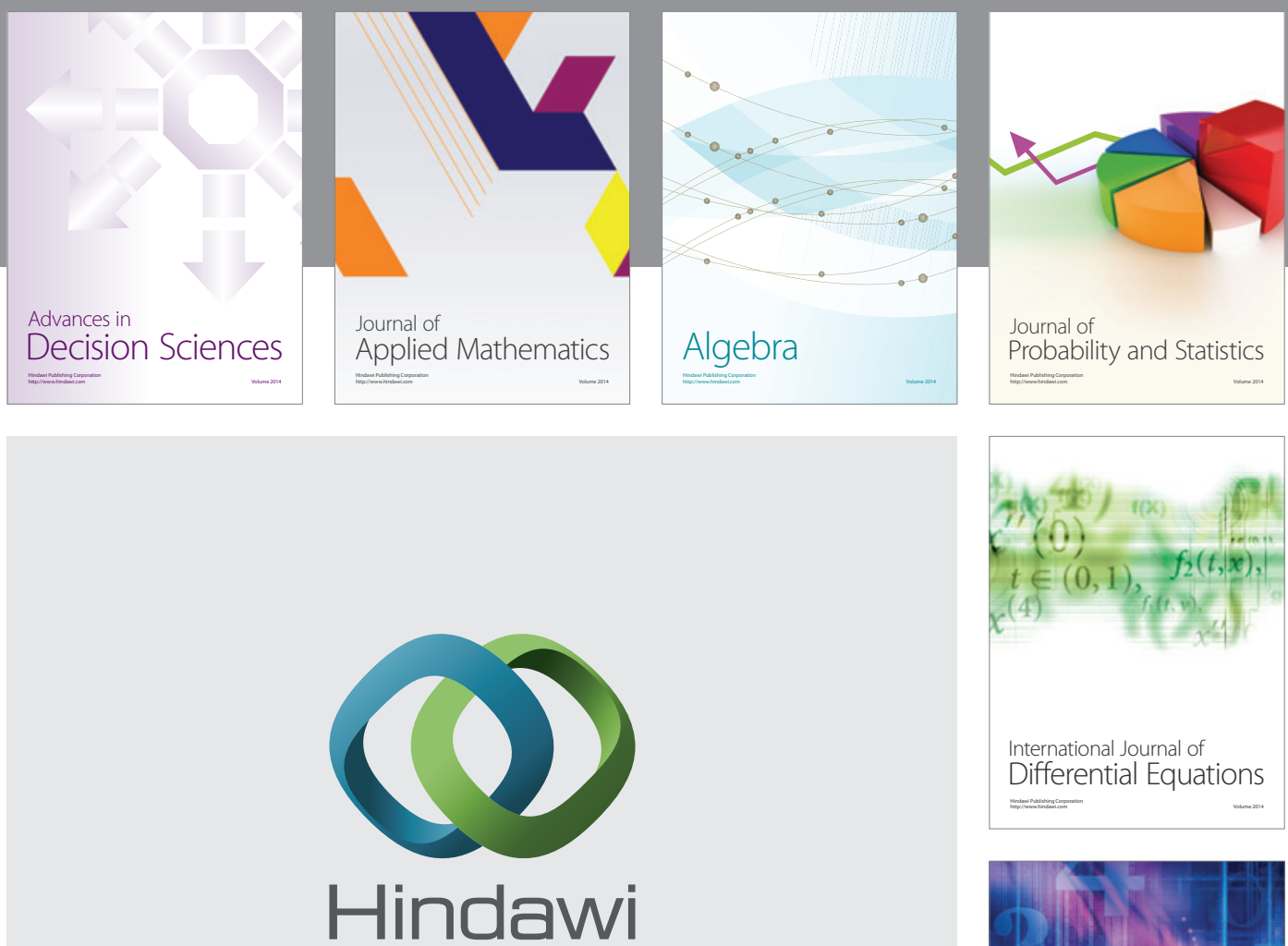

Submit your manuscripts at http://www.hindawi.com
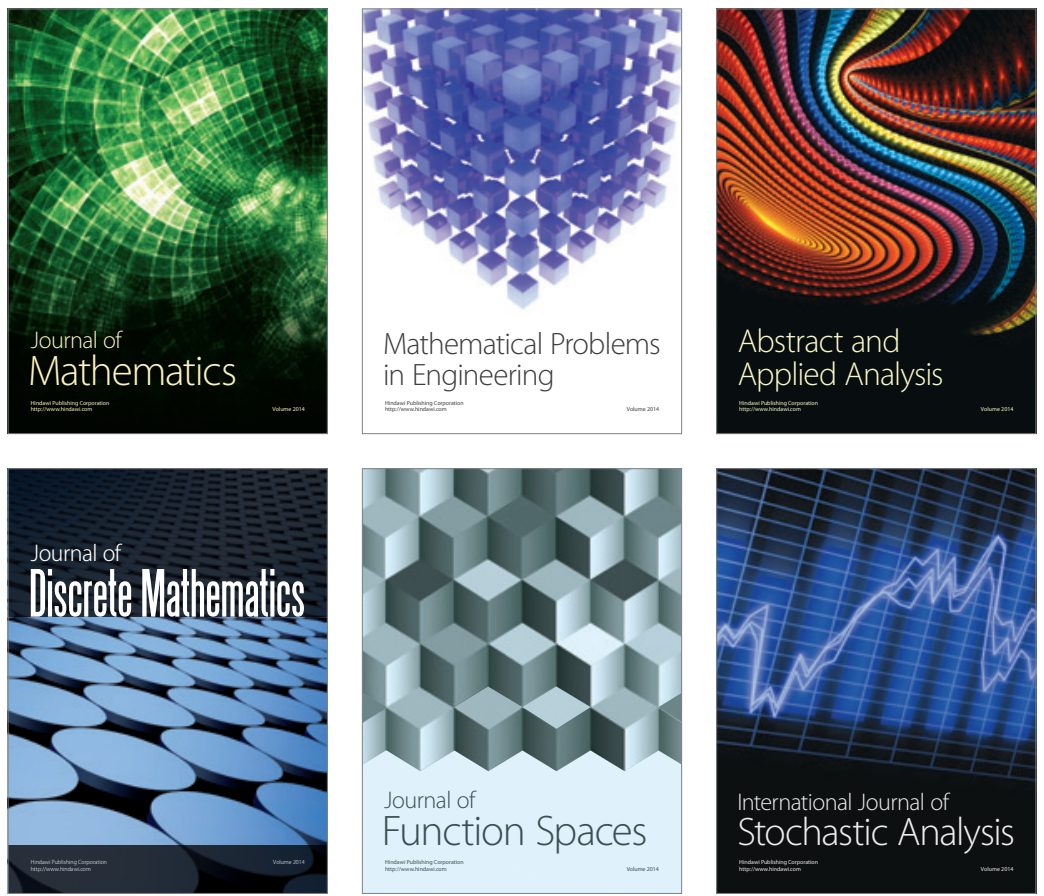

Journal of

Function Spaces

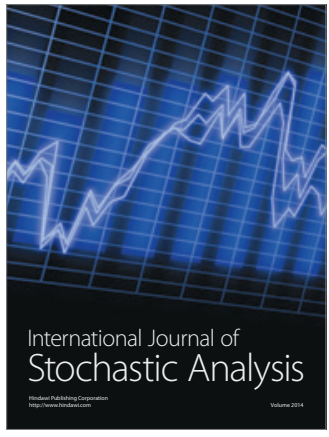

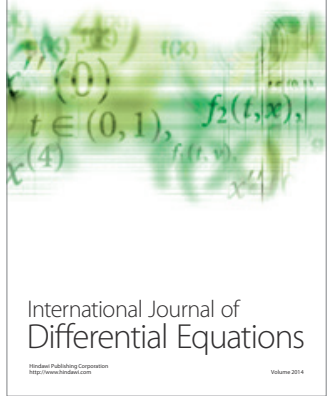
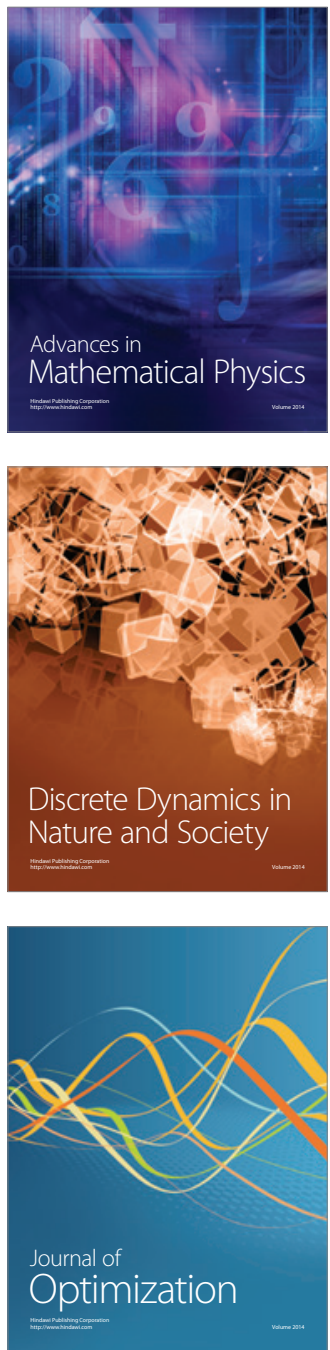